\title{
Torsión de epiplón: \\ causa rara de dolor abdominal recurrente
}

\author{
Omental torsion: a rare cause of recurrent abdominal pain
}

\author{
José Luis Bizueto-Monroy,* Brenda Ivette Montoya-Pérez,** \\ Fernando Saldaña-Torres***
}

Palabras clave: Dolor abdominal, epiplón, tomografía computada.

Key words: Abdominal pain, omentum, computed tomography.

\footnotetext{
* Residente de tercer año de Cirugía General.

** Residente de primer año de Cirugía General. *** Adscrito del Servicio de Cirugía General.
}

Hospital Regional de Petróleos Mexicanos, Salamanca, Guanajuato.

Recibido: 16/12/2015 Aceptado: 27/10/2017

\section{RESUMEN}

Introducción: La torsión de epiplón es una condición en la cual un pedículo del mismo gira sobre su propio eje, lo que produce inflamación por contigüidad e imita los síntomas y signos clínicos de la apendicitis aguda, lo cual puede provocar un diagnóstico erróneo si esta patología no es sospechada. Método: Reportamos un caso de torsión de epiplón con resolución quirúrgica; enfatizamos la presentación clínica, así como los distintos criterios para el abordaje médico o quirúrgico. Reporte de caso: Presentamos el caso de un paciente masculino de 41 años de edad en el Hospital Regional de Petróleos Mexicanos en Salamanca, Guanajuato, con dolor abdominal de más de dos semanas de evolución, con múltiples visitas al Departamento de Urgencias y características clínicas similares a la enfermedad ácido péptica; debido al empeoramiento de su sintomatología, se decidió una exploración quirúrgica de manera laparoscópica, con evidencia de torsión de epiplón en su pedículo, el cual fue resecado sin incidentes; presentó una rápida recuperación, sin complicaciones perioperatorias. Conclusión: La torsión de epiplón es rara; sin embargo, el conocimiento de este tipo de patología es de importancia para el cirujano, ya que su presentación puede ser similar a otras causas de abdomen quirúrgico. El abordaje laparoscópico es una excelente opción para su manejo.

\section{ABSTRACT}

Introduction: Torsion of the greater omentum is a condition in which a pedicle twists on its axis and produces inflammation by contiguity that can mimic the clinical symptoms and signs of acute appendicitis and may lead to a misdiagnosis if it is not clinically suspected. Method: We present a case of a primary torsion of the greater omentum; we emphasize the clinical presentation and different criteria of medical or surgical approach. Case report: We report the case of a patient with a history of more than two weeks of abdominal pain similar to peptic ulcer disease and multiple Emergency Department visits; the symptomatology progressively intensified, so the decision was made to perform laparoscopic surgical exploration that revealed omental torsion, which appeared to have twisted on its pedicle; it was resected, with a rapid and uneventful recovery. Conclusion: Omental torsion is rare; however, the knowledge of this pathology is important to the surgeon because it mimics the common causes of surgical abdomen. Laparoscopic resection is an excellent alternative method of treatment.

\section{INTRODUCCIÓN}

$\mathrm{A}^{\mathrm{p} s \mathrm{~s}^{2}}$ pesar de sus primeras descripciones desde 1899 por Eitel, ${ }^{1}$ la torsión de epiplón es conocida como una entidad poco común dentro de los diagnósticos diferenciales de abdomen quirúrgico, no solo por su baja prevalencia como sospecha diagnóstica, sino también en relación con su cuadro clínico variado: desde apendicitis y peritonitis hasta eventos insidiosos; tal falta de especificidad clínica ha permitido únicamente una certeza diagnóstica prequirúrgica de entre 0.6 y $4.8 \%{ }^{2}$

\section{REPORTE DE CASO}

Presentamos el caso de un paciente masculino de 41 años de edad sin antecedentes de importancia, sedentario, obeso, que acudió al Servicio de Urgencias por dolor abdominal de 12 horas de evolución en el epigastrio postprandial, ardoroso; sin alteraciones en los signos vitales ni paraclínicos. Se le otorgó manejo por probable enfermedad ácido péptica a base de inhibidor de bomba en el Servicio de Urgencias. Posteriormente, presentó un cuadro clínico similar a los tres días; en esta 
ocasión, se realizó ultrasonografía abdominal, que no mostró alteraciones estructurales, por lo que se continuó con el manejo médico previo en el Servicio de Gastroenterología. A las dos semanas del último evento, recurrió la sintomatología; en esta ocasión, con irradiación al flanco derecho, acompañándose de náuseas, malestar general, signos vitales con taquicardia de 110 latidos por minuto y fiebre de 38.5 grados centígrados. A la exploración física, se observó paciente con obesidad grado II, a la palpación con presencia de McBurney, Von Blumberg y Rovsing. Los exámenes de laboratorio, con biometría hemática dentro de los parámetros normales, a excepción por leucocitosis $\left(11,000 / \mathrm{mm}^{3}\right)$. Se realizó tomografía simple de abdomen, con patrón de estrías y curvas dependiente de epiplón mayor en el flanco derecho, sin lograr visualizar el apéndice (Figuras 1 y 2). Debido a la recurrencia de la sintomatología, el empeoramiento del cuadro clínico, con presencia de respuesta inflamatoria sistémica, se decidió un manejo quirúrgico con abordaje laparoscópico; se halló porción derecha del epiplón mayor de aproximadamente $6 \times 2 \times 0.5 \mathrm{~cm}$, gangrenosa, rotada en su eje con sentido contrario a las manecillas del reloj, con escaso líquido inflamatorio serohemático (Figura 3), la cual se decidió resecar con engrapadora laparoscópica. Se encontró el apéndice cecal macroscópicamente normal; se realizó apendicectomía con engrapadora laparoscópica de igual forma. Cursó estancia

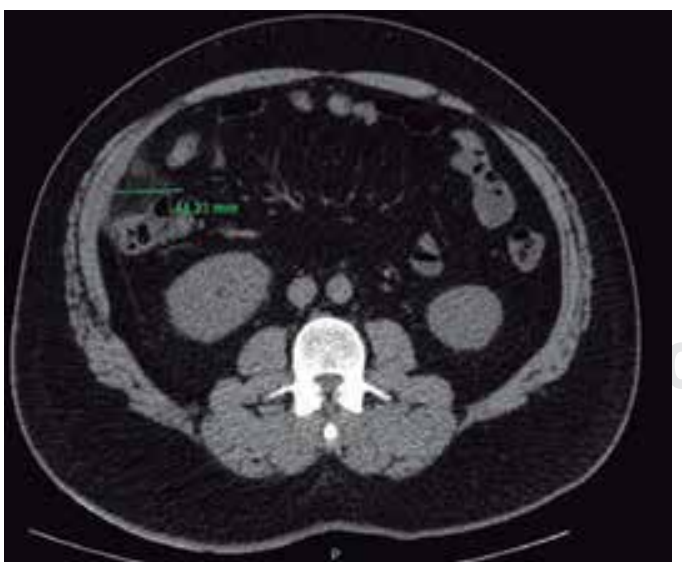

Figura 1. Tomografía axial simple con patrón de estrías hiperdensas, concéntricas, en el flanco derecho. hospitalaria sin complicaciones y egresó a las 24 horas postquirúrgicas.

\section{DISCUSIÓN}

La torsión de epiplón es una condición patológica en la cual un pedículo del mismo puede girar a lo largo de su eje, con compromiso vascular y necrosis del segmento afectado; puede presentarse con una gran variedad de sintomatología y ser confundido muchas veces con apendicitis, colecistitis, diverticulitis aguda, divertículo de Meckel. ${ }^{3}$ Comparado con estas patologías, posee una incidencia menor de aproximadamente $0.0016-0.37 \% .{ }^{4}$ A pesar de

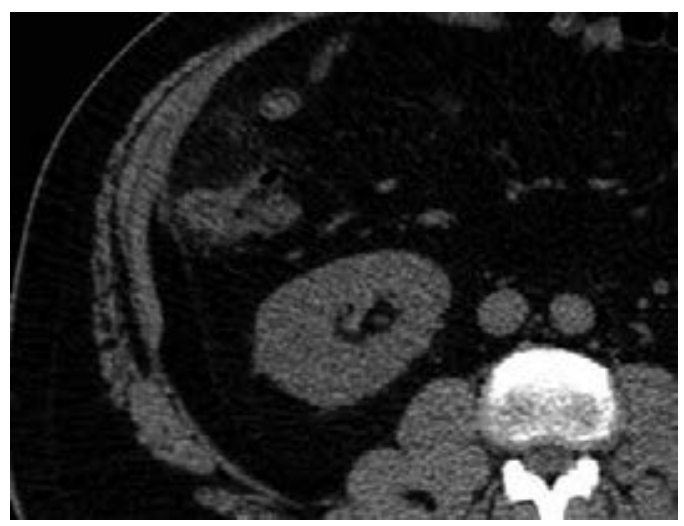

Figura 2. Aspecto de conglomerado epiploico por cambios heterogéneos en la densidad y concéntricos en el flanco derecho.

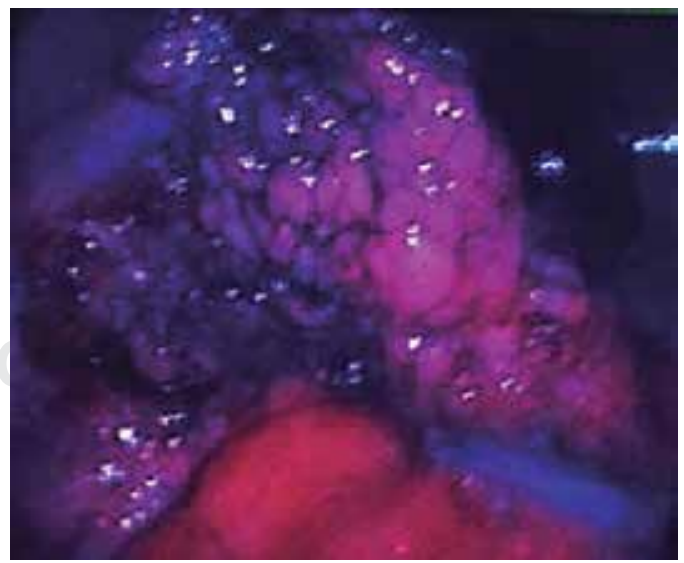

Figura 3. Porción del epiplón mayor rotada en su pedículo, con compromiso vascular evidente. 
que su patogénesis aún no se define claramente, se han encontrado factores predisponentes, como el incremento de la presión abdominal, el ejercicio intenso, la obesidad (índice de masa corporal $>30 \mathrm{~kg} / \mathrm{m}^{2}$ ), hiperperistalsis, trauma, variaciones del omento (como anatomía bífida y pedículo estrecho). ${ }^{5,6}$ La torsión del epiplón puede clasificarse como primaria (la cual es más habitual en edad pediátrica, sin causa orgánica aparente) y secundaria (relacionada con otra patología abdominal, como quiste de mesenterio, tumoraciones, hernias de pared abdominal o adherencias). ${ }^{7}$

La mayoría de los casos ocurren en personas adultas y de género masculino, aunque también se encuentra en edad pediátrica, en un 15\% aproximadamente. Su presentación clínica más frecuente es el dolor abdominal indistinguible con apendicitis, acompañado de síntomas constitucionales. Por lo anterior, los estudios de imagen como el ultrasonido poseen un rol de gran importancia para el diagnóstico diferencial y, a pesar de su baja sensibilidad, posee alta especificidad para poder descartar patologías más comunes de origen ginecológico o apendicitis. Los hallazgos más frecuentes son tumoración no compresible, hiperecoica, en el cuadrante superior derecho, adherente a la pared abdominal anterior, con visualización de apéndice cecal normal o sin visualización del mismo. ${ }^{8,9}$ En las distintas referencias de nuestra revisión no se ha reportado epigastralgia ni enfermedad ácido péptica como diagnóstico diferencial en este tipo de condiciones. En nuestro caso, fue la sintomatología predominante y un ultrasonido abdominal sin alteraciones lo que condujo a la falta de sospecha clínica.

Empero, el incremento de la disponibilidad y el uso de la tomografía computada ha aumentado la certeza quirúrgica antes del procedimiento, con una elevada sensibilidad; los hallazgos pueden ser una tumoración heterogénea, concéntrica, de densidad grasa, localizada entre transverso e hígado, con cambios inflamatorios en su periferia, aunque dichas modificaciones podrían no ser notorias si el eje de rotación no se encuentra perpendicular al plano de la imagen. ${ }^{8,9}$ Otra herramienta de imagen -aunque no ampliamente utilizada por su disponibilidad - es la resonancia magnética, que puede mostrar un patrón lineal hi- pointenso en las estructuras adyacentes en T1, así como una tumoración hiperintensa en T2. ${ }^{9}$

La mayoría de la literatura recomienda un manejo médico primario a base de antimicrobianos y antiinflamatorios no esteroideos, ${ }^{10}$ debido a su curso benigno y autolimitado, con un periodo de resolución de alrededor de dos semanas. Se ha sugerido un manejo quirúrgico únicamente en casos con complicaciones como formación de abscesos o hemoperitoneo. De realizarse, se recomienda apendicectomía en el mismo tiempo quirúrgico para evitar confusiones diagnósticas a futuro, incluyendo una posible recurrencia de torsión omental.6,8,11 En el caso reportado, se optó por la resolución quirúrgica debido al tiempo de evolución, recurrencia, valoraciones previas de distintos servicios y manifestaciones de respuesta inflamatoria sistémica.

Aunque no existe evidencia significativa para demostrar el pronóstico en relación con el tratamiento médico, se sugiere vigilancia al menos entre 24 y 48 horas en hospitalización, y de ser necesario, considerar el manejo quirúrgico ante el deterioro clínico o en marcadores de laboratorio e imagen. ${ }^{12}$

\section{CONCLUSIONES}

La torsión de epiplón primaria debe ser considerada como diagnóstico diferencial ante sospecha de apendicitis aguda en casos donde la clínica, laboratorios, imagen y hallazgos transquirúrgicos no correlacionan entre sí.

Por lo anterior, a pesar de su baja incidencia, el diagnóstico prequirúrgico de esta patología podrá ser más frecuente ante el incremento del uso de herramientas diagnósticas de imagen como el ultrasonido y/o la tomografía computada, y con ello, será posible un manejo conservador de primera instancia. Por otra parte, la laparoscopia diagnóstica continuará siendo el recurso diagnóstico-terapéutico de elección ante la falta de dichas herramientas, presencia de complicaciones y baja sospecha diagnóstica de esta patología.

\section{Agradecimientos}

Nos gustaría agradecer a Giovanna Domínguez Hernández por su apoyo incondicional y al 
equipo quirúrgico del Hospital Regional de Petróleos Mexicanos de Salamanca, Guanajuato por su cooperación durante el procedimiento quirúrgico.

\section{Aspectos éticos}

El consentimiento informado firmado por el paciente y testigos se encuentra asentado en el expediente clínico del hospital.

\section{REFERENCIAS}

1. Eitel GG. Rare omental torsion. New York Rec. 1899; 55: 715-716.

2. Tsironis A, Zikos N, Bali C, Pappas-Gogos G, Koulas S, Katsamakis N. Acute abdomen due to primary omental torsion: case report. J Emer Med. 2011; 79: e55-e58.

3. Andreuccetti J, Ceribelli C, Manto O, Chiaretti, Negro P, Tuscano D. Primary omental torsion (POT): a review of literature and case report. World J Emerg Surg. 2011; 6: 6.

4. Benaghmouch F, Aalala EM, Hrora A, Benamer A, Sabbah F, Ahallat $M$ et al. Acute abdomen for omental torsion. European J Radiol Extra. 2011; 79: e55-e57.

5. García E, Robles P. Torsión omental secundaria a hernia inguinal. Radiología. 2015; 2: 173-178.

6. Abidin AZ, Rahmat K, Aziz A. Right-sided omental torsion associated with inguinal hernia: $\mathrm{CT}$ imaging features. European J Radiol Extra. 2007; 64: 23-25.
7. Chew KD, Holgersen LO, Friedman D. Primary omental torsion in children. J Ped Surg. 1995; 30: 816-817.

8. Nubi A, McBride W, Stringel G. Primary omental infarct: conservative vs operative management in the era of ultrasound, computerized tomography, and laparoscopy. J Ped Surg. 2009; 44: 953-956.

9. Lee RK-I, Chu WC-w. Omental torsion in children successfully treated by conservative treatment based on radiological diagnosis. Eur J Radiol Extra. 2010; 75: e75-e77.

10. Hsu BC-H, Chou DA. Primary idiophatic segmental infarction of the greater omentum. FJS. 2011; 44: 233-236.

11. Abe T, Kajiyama K, Harimoto N, Gion T, Nagaie T. Laparoscopic omentectomy for preoperative diagnosis of torsion of the greater omentum. Int J Surg Case Rep. 2012; 3: 100-102.

12. Barai KP, Knight BC. Diagnosis and management of idiopathic omental infarction: a case report. Int J Surg Case Rep. 2011; 2: 138-140. 